\title{
Characteristics of
}

Carbapenemase-Producing Klebsiella pneumoniae Isolated in the Intensive Care Unit of the Largest Tertiary Hospital in Bangladesh

Takashi Okanda',2,3*, Anwarul Haque ${ }^{3}$, Takuro Koshikawa ${ }^{2}$, Amirul Islam $^{4}$, Qumrul Huda $^{4}$,
Hiromu Takemura

${ }^{1}$ Department of Microbiology, Tokyo Medical University, Tokyo, Japan, ${ }^{2}$ Department of Microbiology, St. Marianna University School of Medicine, Kawasaki, Japan, ${ }^{3}$ Department of Infectious Diseases, International University of Health and Welfare, Narita, Japan, ${ }^{4}$ Department of Anesthesia, Analgesia and Intensive Care Medicine, Bangabandhu Sheikh Mujib Medical University, Dhaka, Bangladesh

For addressing the issue of antimicrobial drug resistance in developing countries, it is important to investigate the characteristics of carbapenemase-producing organisms. We aimed to genetically characterize a carbapenemase-producing Klebsiella pneumoniae (CPKP) isolated in the intensive care unit of a tertiary hospital in Bangladesh. The number of CPKP isolates were 43/145 (30\%), of which pandrugresistant (PDR) strains were 14\%. These carbapenemases were New Delhi metallobeta-lactamase (NDM)-1 (53\%), NDM-5 (14\%), oxacillinase (OXA)-181 (12\%), OXA232 (10\%), NDM-5 + OXA-181 (5\%), and NDM-5 + OXA-232 (2\%). Many CPKP isolates harbored a variety of resistance genes, and the prevalence of $16 \mathrm{~S}$ rRNA methyltransferase was particularly high (91\%). The 43 CPKP isolates were classified into 14 different sequence types (STs), and the common STs were ST34 (26\%), ST147 (16\%), ST11 (9\%), ST14 (9\%), ST25 (7\%), and ST231 (7\%). In this study, PDR strains were of three types, ST147, ST231, and ST14, and their PDR rates were 57, 33, and 25\%, respectively. The spread of the antimicrobial drug resistance of CPKP in Bangladesh was identified. In particular, the emergence of PDR is problem, and there may be its spread as a superbug of antimicrobial treatment.

Keywords: PDR, ST147, NDM-1, NDM-5, OXA-181, OXA-232

\section{INTRODUCTION}

The worldwide spread of carbapenemase-producing organisms (CPOs) is increasing (Bonomo et al., 2009). Carbapenem resistance in Klebsiella pneumoniae is frequently associated with antimicrobial resistance to other antibiotic classes, making treatment of infections challenging (Fournier et al., 2018). In North America, South America, and Europe, the main carbapenemaseproducing genes are the Klebsiella pneumoniae carbapenemase (KPC) types; in the Middle East and Africa, they are the oxacillinase-48-like (OXA-48-like) types; and in the Indian subcontinent, they are of the New Delhi metallo-beta-lactamase (NDM) and OXA-48-like types. Many of these multidrug-resistant (MDR) strains are sequence type (ST) 11, ST14, ST37, ST147, and ST258 and are spreading worldwide as international MDR high-risk clones (Mathers et al., 2015). 
The Center for Disease Control and Prevention (CDC) and the European Centre for Disease Prevention and Control have jointly developed definitions for MDR, extensively drug-resistant (XDR), and pandrug-resistant (PDR) bacteria (Magiorakos et al., 2012). K. pneumoniae strains classified as XDR are rapidly emerging due to the dissemination of resistance toward aminoglycosides, $\beta$-lactams, fluoroquinolones, and carbapenems (Al-Marzooq et al., 2015). Recently, XDR strains have evolved to become PDR by acquiring resistance to tigecycline and polymyxin antibiotics (Papadimitriou-Olivgeris et al., 2018). The spread of such strains is associated with high mortality rates, limited treatment options, and rapid dissemination of successful bacterial clones in the hospital setting (Guo et al., 2016; Protonotariou et al., 2018).

Antimicrobial resistance (AMR) is a major problem in Asia (O'neill, 2016). Bangladesh is a developing country surrounded by India and is known to have a high prevalence of carbapenemresistant organisms. The CPOs detected are mainly NDMproducing strains, but in recent years, OXA-48-like-producing strains have also been detected. However, no surveillance system has been established in developing countries in this region, and there are few reports on molecular epidemiology (Hasan and Rabbani, 2019). We started the short-term prospective study of MDR bacteria with the ultimate goal of building a resistant surveillance system in Bangladesh (Okanda et al., 2018). In this study, we performed an analysis of acquired drug resistance mechanism and molecular epidemiology of carbapenemaseproducing Klebsiella pneumoniae (CPKP) isolated in a tertiary hospital in Dhaka, Bangladesh.

\section{MATERIALS AND METHODS}

\section{Collection of Isolates}

Bangabandhu Sheikh Mujib Medical University Hospital is the premier Postgraduate Medical Institution of Bangladesh and a major tertiary hospital with 1,900 beds. The intensive care unit (ICU) at this hospital accepts critically ill patients not only throughout Dhaka but also from neighboring countries. In this study, collection of isolates was originally scheduled for only between August and October 2015, but because of observed subsequent changes in AMR at ICU, isolates were also collected between August and September 2017. A total of 145 thirdgeneration cephalosporin-resistant Klebsiella pneumoniae were isolated from blood, sputum or tracheal aspirate, urine, and pus in ICU patients (82 in 2015 and 63 in 2017). These were all consecutive isolates selected on the CHROMagar ESBL medium (Kanto Chemical Co., Inc., Japan), eliminating duplication by patient. These isolates were inoculated into casitone medium and transported to Tokyo Medical University in Japan, where they were incubated on modified Drigalski agar (EIKEN Chemical Co., Ltd) at $35^{\circ} \mathrm{C}$ for $18 \mathrm{~h}$. The resulting cultures were reidentified by matrix-assisted laser desorption/ionization timeof-flight mass spectrometry using MALDI Biotyper (library ver. 9.0.0.0) and stored at $-80^{\circ} \mathrm{C}$ in heart infusion broth containing $20 \%$ glycerol until use.

\section{Screening of Carbapenemase-Producer and Detection of Carbapenemase-Encoding Genes}

Phenotypic detection of carbapenemase was performed using the modified carbapenem inactivation method (mCIM) and Carba NP test. Escherichia coli ATCC 25922 was used as an indicator organism and negative control, whereas K. pneumoniae KPP 127 was used as a positive control. All isolates that showed positive results and intermediate results were detected of carbapenemase-encoding genes by polymerase chain reaction (PCR) assay. DNA was extracted using the Cica Geneus DNA Extraction Reagent (Kanto Chemical Co., Inc., Japan). The examined carbapenemase-encoding genes were $b l a_{K P C}, b l a_{G E S}$, bla $a_{I M P}, b l a_{V I M}, b l a_{N D M}$, bla $a_{O X A-48-l i k e}, b l a_{B I C}, b l a_{A I M}, b l a_{G I M}$, bla $a_{S I M}, b l a_{D I M}$, and $b l a_{S P M}$ (Supplementary Table S1).

\section{Genotypic Detection of Carbapenemase-Encoding Genes and Other Resistance Genes}

The confirmed CPKP isolates were tested for extended-spectrum $\beta$-lactamases (ESBLs)-producing genes, plasmid-mediated AmpC $\beta$-lactamase (PABLs)-producing genes, mobilized colistin resistance $(\mathrm{mcr})$ gene, $16 \mathrm{~S}$ rRNA methyltransferase (16SRMTase) genes, tetracycline resistance genes, plasmidmediated quinolone resistance (PMQR) genes, fosfomycin resistance genes, sulfonamide resistance genes, and dihydrofolate reductase (DHFR)-encoding genes (Supplementary Table S1). Target genes were confirmed via single PCR, and PCR fragments were then purified from PCR product or agarose gels using Cica Geneus ${ }^{\circledR}$ PCR \& Gel Prep Kit (Kanto Chemical Co., Inc., Japan). Following sequencing, the similarity search was performed with the sequenced data using BLAST analysis ${ }^{1}$.

\section{Antimicrobial Susceptibility Testing}

Carbapenemase-producing Klebsiella pneumoniae isolates were tested for their susceptibility to piperacillin/tazobactam (TZP), cefepime (FEP), cefmetazole (CMZ), moxalactam (MOX), flomoxef (FOX), aztreonam (ATM), imipenem (IPM), meropenem (MEM), doripenem (DOR), biapenem (BPM), colistin (CST), gentamicin (GEN), amikacin (AMK), minocycline (MIN), tigecycline (TGC), ciprofloxacin (CIP), levofloxacin (LVX), fosfomycin (FOF), and trimethoprim/sulfamethoxazole (SXT). Microdilution susceptibility testing was performed and minimum inhibitory concentrations (MIC) determined according to the Clinical and Laboratory Standards Institute (CLSI) guidelines (Clinical and Laboratory Standards Institute (CLSI), 2020). In this edition, CST breakpoints were defined as resistant $\geq 4 \mathrm{mg} / \mathrm{l}$ and intermediate $\leq 2 \mathrm{mg} / \mathrm{l}$, with no susceptible category defined. In addition, no TGC and FOF breakpoints were defined for $K$. pneumoniae. Thus, CST, TGC, and FOF breakpoints were performed according to the European Committee on Antimicrobial Susceptibility Testing

\footnotetext{
${ }^{1}$ http://blast.ncbi.nlm.nih.gov/Blast.cgi
} 
(EUCAST) guideline ${ }^{2}$. E. coli ATCC 25922 was used for quality control of the test.

\section{Molecular Epidemiology Typing}

Pulsed-field gel electrophoresis (PFGE) was carried out by the following method. After digestion of the genomic DNA by XbaI (New England Biolabs), the restriction fragments were separated using a temperature-controlled CHEF-DR III system (Bio-Rad) for $19.5 \mathrm{~h}$ at $14^{\circ} \mathrm{C}$ under the following conditions: two state modes, initial switch time of $5.3 \mathrm{~s}$, final switch time of $60.0 \mathrm{~s}$, a gradient of $6 \mathrm{~V} / \mathrm{cm}$, and switch angle of $120^{\circ}$. After staining with ethidium bromide, the fragments were visualized using an ultraviolet transilluminator. Percent similarities were determined by generating a dendrogram by the unweighted pair group method using arithmetic averages and based on Dice coefficients. Band position tolerance and optimization were both set at $1.0 \%$. A similarity coefficient of $80.0 \%$ was selected to define the clusters after reviewing the epidemiological data associated with each isolate cluster. For the determination of PCR conditions and primer sequences, information obtained from the Institut Pasteur multilocus sequence typing (MLST) database ${ }^{3}$ was used (Diancourt et al., 2009). For the identification of ST, the allelic profiles of the purified sequences were submitted to the Institut Pasteur MLST database. Clustering of each ST to determine the clonal group (CG) of single-locus mutants was conducted using the eBURST program (Feil et al., 2004).

\section{RESULTS}

\section{CPKP Isolates}

By phenotypic testing and sequencing, carbapenemaseproducing genes were detected from 43 of the 145 isolates (30\%). These 43 carbapenemase-producing genes were identified as bla $_{N D M-1}(23,53 \%), b l a_{N D M-5}(6,14 \%), b l a_{O X A-181}$ (5, $12 \%)$, bla OXA-232 $(4,10 \%), 2$ bla $a_{N D M-5}+$ bla $a_{O X}-181$ (2, $5 \%), b l a_{N D M-5}+b_{0 X A-232}(1,2 \%), b l a_{N D M-7}(1,2 \%)$, and bla $a_{O X A-48}(1,2 \%)$ by sequence. Therefore, $40(93 \%)$ of the 43 CPKP isolates were single-harboring $b a_{N D M}(30,70 \%)$ or bla OXA-48-like $(10,23 \%)$, while the remaining $3(7 \%)$ were coharboring both genes. CPKP isolates were co-harboring various AMR genes (Figure 1). ESBL- and PABL-encoding genes were detected in $43(100 \%)$ and 22 (51\%) CPKP isolates, respectively. Therefore, 22 CPKP isolates (51\%) were co-harboring ESBLs and PABLs. In addition, the prevalence of 16SRMTase, tetracycline resistance, $\mathrm{PMQR}$, fosfomycin resistance, sulfonamide resistance, and DHFR-encoding genes in CPKP isolates were 91\% (39), 30\% (13), $77 \%(33), 49 \%(21), 60 \%(26)$, and $60 \%$ (26), respectively. However, the $\mathrm{mcr}$ gene reported to date was not detected from colistin-resistant isolates.

\section{Antimicrobial Resistance}

The susceptibility of 43 CPKP isolates are shown in Table $\mathbf{1 .}$ Forty-three CPKP isolates were resistant to many of the

\footnotetext{
${ }^{2}$ https://eucast.org/clinical_breakpoints/

${ }^{3}$ https://bigsdb.pasteur.fr/klebsiella/klebsiella.html
}

antibiotics tested, with 10 isolates (23\%) classified as XDR and 6 isolates (14\%) classified as PDR. Interestingly, these CPKP isolates were highly resistant to aminoglycosides, and all AMKresistant isolates showed MIC $\geq 512$. From 2015 to 2017, the resistant rates of BPM, MIN, TGC, LVX, FOF, and SXT were increased, while the CST susceptibility was almost unchanged. In addition, the MIC value of six PDR isolates are shown in Table 2. PDR isolates showed high-level resistance to many antimicrobials, but low-level resistance to CST and TGC.

CTX-M-15 was the common ESBL, but PABL was changed from DHA to CMY in 2017 (Figure 2). In 16SRMTase, the prevalence did not change during the collection period, while the common type was changed from the armA gene to the $r m t B / F$ genes. The 2017 CPKP isolate showed an increased prevalence of some resistance genes compared to the 2015 isolate. In tetracycline resistance, the prevalence of the tet $(A)$ gene increased from $5 \%(1 / 21)$ to $36 \%(8 / 22)$. Perhaps this is the cause of the increased MINO or TGC resistance rate. In addition, the prevalence of the fos $A$, sull, and $d f r A 1$ genes were increased from $29 \%(6 / 21)$ to $68 \%(15 / 22)$, from $29 \%(6 / 21)$ to $86 \%(19 / 22)$, and from $19 \%(4 / 21)$ to $73 \%(16 / 22)$, respectively. As the number of these resistance genes increased, the resistance rate of the corresponding antibacterial drug increased. In fact, the increase in resistant rates from 2015 to 2017 was $29 \%(6 / 21)$ to $68 \%$ (15/22) for FOF and 33\% (7/21) to 86\% (19/22) for SXT.

\section{Molecular Epidemiological Characteristics of CPKP}

Pulsed-field gel electrophoresis identified 29 different banding patterns from 43 CPKP isolates. These banding patterns were classified into 18 clusters (A to R) by more than $80 \%$ genetic associations (Figure 1). Common clusters were R (11, 26\%), A (4, 9\%), and $\mathrm{L}(4,9 \%)$, and the most common cluster $\mathrm{R}$ was all ST34 strain. All ST34s were isolated from the ICU in 2015, and their antimicrobial susceptibility and acquisition resistance genes were almost the same. This suggested that there was an outbreak of ST34 strains in 2015. The STs of clusters A and L were ST147 and ST11, respectively. On the other hand, the second most common cluster A was all ST147, but it may be a different clone due to different banding patterns and separation dates. The band pattern of ST147 was significantly different between 2015 and 2017, forming cluster A and B, respectively. Therefore, it was considered that the transmission route was different between the ST147 strain in 2015 and in 2017.

The MLST classified the 43 isolates into 14 different STs (Figure 1). The common STs were ST34 (11, 26\%), ST147 (7, 16\%), ST11 (4, 9\%), ST14 (4, 9\%), ST25 (3, 7\%), and ST231 (3, 7\%). ST147, ST11, and ST231 strains were isolated in both 2015 and 2017. The eBURST analysis, based on the MLST database, found that 14 different STs were clustered into four different CGs (Figure 3). The founder of each CG was ST11, ST147, ST231, and ST43, while ST893 did not form a cluster and was a singleton. CG11 was the most common group, but those STs were diverse, and only ST11 strain was isolated in both years. The multidrug resistance of ST147 and ST231 strains was serious, all of which were XDR or PDR. 


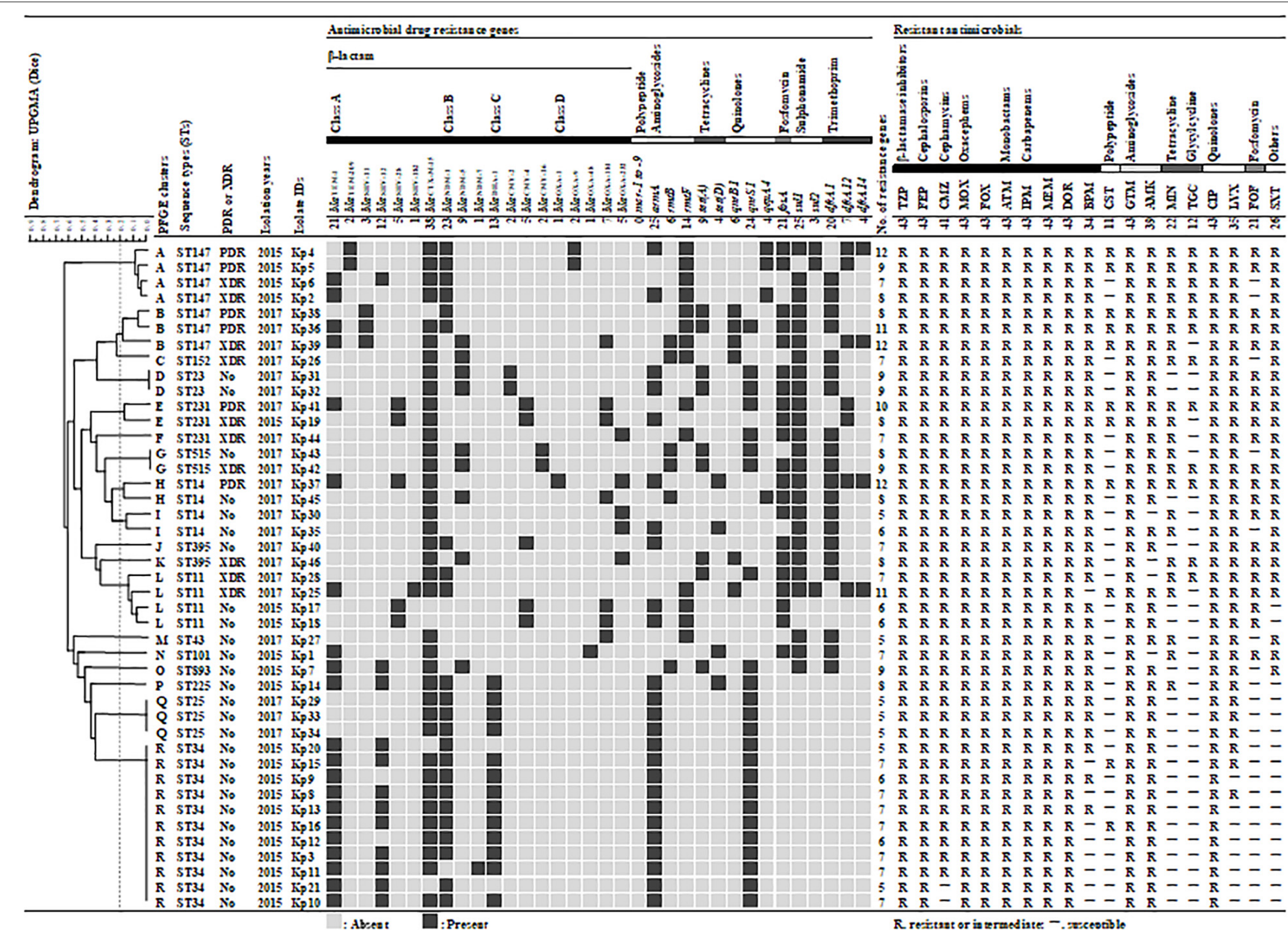

FIGURE 1 | Genetic and antibiogram profiles of 43 carbapenemase-producing Klebsiella pneumoniae isolates. A similarity coefficient of $80 \%$ was selected to define the clusters after reviewing the epidemiological data associated with each of the clusters of isolates. The presence or absence of plasmid-mediated antimicrobial resistance genes is indicated by black filled square or gray filled square, respectively. Antimicrobial susceptibility: susceptible (S) and resistant/intermediate (-). TZP, piperacillin/tazobactam; FEP, cefepime; CMZ, cefmetazole; MOX, moxalactam; FOX, flomoxef; ATM, aztreonam; IPM, imipenem; MEM, meropenem; DOR, doripenem; BPM, biapenem; CST, colistin; GEN, gentamicin; AMK, amikacin; MIN, minocycline; TGC, tigecycline; CIP, ciprofloxacin; LVX, levofloxacin; FOF, fosfomycin; SXT, trimethoprim/sulfamethoxazole. 
TABLE 1 | Susceptibility of carbapenemase-producing Klebsiella pneumoniae in Bangladesh.

\begin{tabular}{|c|c|c|c|}
\hline & 2015 & 2017 & Total \\
\hline Number of isolates & $21 / 82(26 \%)$ & 22/63 (35\%) & $43 / 145(30 \%)$ \\
\hline \multicolumn{4}{|l|}{ Antimicrobial resistance types } \\
\hline Multidrug resistant & $16 / 21(76 \%)$ & $11 / 22(50 \%)$ & $27 / 43(63 \%)$ \\
\hline Extensively drug resistant & $3 / 21(14 \%)$ & $7 / 22(32 \%)$ & $10 / 43(23 \%)$ \\
\hline Pandrug resistant & $2 / 21(10 \%)$ & $4 / 22(18 \%)$ & $6 / 43(14 \%)$ \\
\hline \multicolumn{4}{|l|}{ Resistant rate } \\
\hline Piperacillin-tazobactam & $100 \%$ & $100 \%$ & $100 \%$ \\
\hline Cefepime & $100 \%$ & $100 \%$ & $100 \%$ \\
\hline Cefmetazole & $90 \%$ & $100 \%$ & $95 \%$ \\
\hline Moxalactam & $100 \%$ & $100 \%$ & $100 \%$ \\
\hline Flomoxef & $100 \%$ & $100 \%$ & $100 \%$ \\
\hline Aztreonam & $100 \%$ & $100 \%$ & $100 \%$ \\
\hline Imipenem & $100 \%$ & $100 \%$ & $100 \%$ \\
\hline Meropenem & $100 \%$ & $100 \%$ & $100 \%$ \\
\hline Doripenem & $100 \%$ & $100 \%$ & $100 \%$ \\
\hline Biapenem & $62 \%$ & $95 \%$ & $79 \%$ \\
\hline Colistin & $24 \%$ & $27 \%$ & $26 \%$ \\
\hline Gentamicin & $100 \%$ & $100 \%$ & $100 \%$ \\
\hline Amikacin & $95 \%$ & $86 \%$ & $91 \%$ \\
\hline Minocycline & $33 \%$ & $68 \%$ & $51 \%$ \\
\hline Tigecycline & $19 \%$ & $36 \%$ & $28 \%$ \\
\hline Ciprofloxacin & $100 \%$ & $100 \%$ & $100 \%$ \\
\hline Levofloxacin & $62 \%$ & $100 \%$ & $81 \%$ \\
\hline Fosfomycin & $29 \%$ & $68 \%$ & $49 \%$ \\
\hline Trimethoprim-sulfamethoxazole & $33 \%$ & $86 \%$ & $60 \%$ \\
\hline
\end{tabular}

Breakpoints are performed according to the Clinical and Laboratory Standards Institute guideline M100-30 edition and according to the European Committee on Antimicrobial Susceptibility Testing guideline ver.10.0 for colistin, tigecycline, and fosfomycin.

\section{DISCUSSION}

The global spread of CPOs is a major issue in AMR measures, and the emergence of PDR strains makes antimicrobial treatment more difficult. The worsening of resistance of K. pneumoniae is remarkable, and some international MDR high-risk clones are reported (Mathers et al., 2015). These clones have a significant burden on global public health. Therefore, it is necessary to conduct intensive studies to monitor K. pneumoniae international MDR high-risk clones, to mitigate this issue. In the present study, we report for the molecular epidemiological characteristics of CPKP isolates collected at the largest tertiary hospital in Bangladesh. It is worth noting that PDR strains are ST147, ST231, and ST14, which harbored various resistance genes

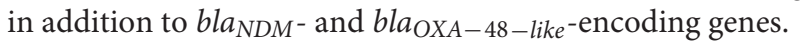

Antimicrobial resistance is globally increasing via the spread of carbapenemase, and treatment options are increasingly limited (Bonomo et al., 2009). In several cases, CPE are also resistant to colistin and tigecycline, and our data agrees with this observation (Table 2). In our study, the colistin resistance rate of CPKP isolates was $26 \%(\mathrm{MIC}=4 \mathrm{mg} / \mathrm{l})$. Colistin resistance is caused by a decrease in negative charge due to modification of the lipid A structure of the outer membrane LPS. Modifications

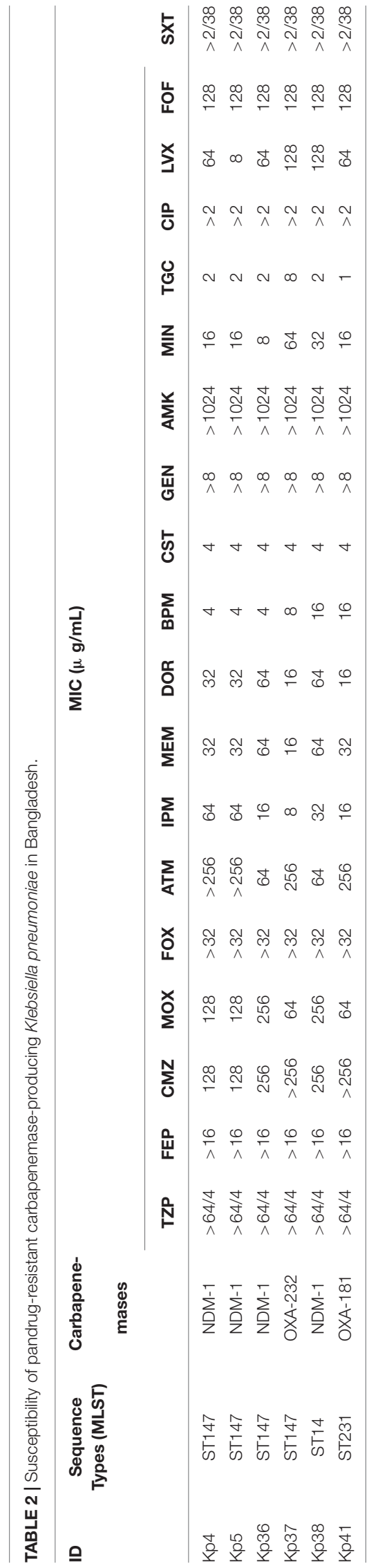


A

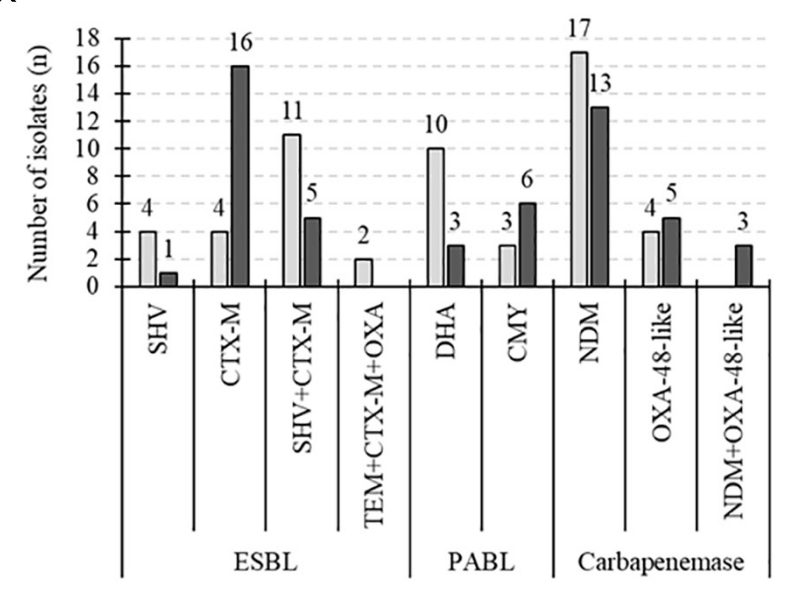

C

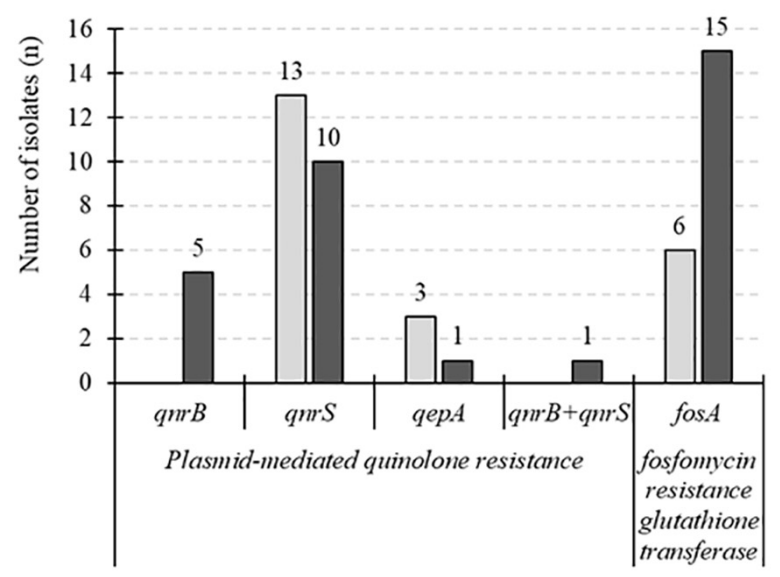

B

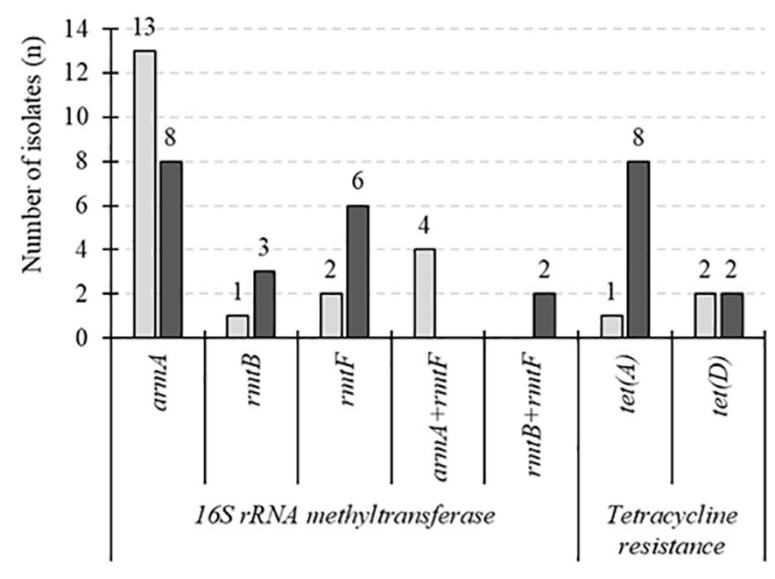

D

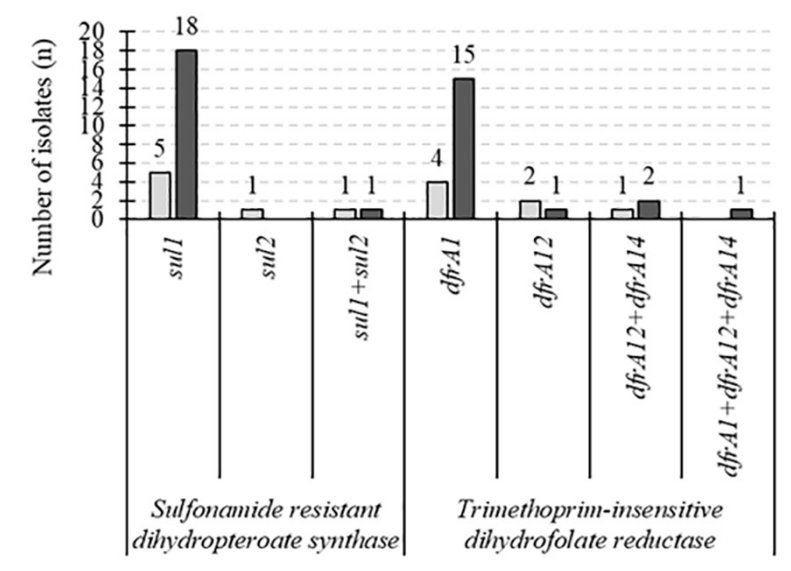

FIGURE 2 | Changes in the number of detected resistance genes. (A) Extended-spectrum $\beta$-lactamases (ESBLs)-, plasmid-mediated AmpC $\beta$-lactamase (PABLs)-, and carbapenemase-producing genes. (B) 16S rRNA methyltransferase- and tetracycline resistance protein-producing genes. (C) Plasmid-mediated quinolone resistance and fosfomycin resistance genes. (D) Sulfonamide resistance genes and dihydrofolate reductase encoding genes.

include those by the acquired $m c r$ gene and those by the twocomponent regulatory system caused by chromosomal gene mutations (Macesic et al., 2020). However, the $\mathrm{mor}$ gene was not detected from CST-resistant isolates. Thus, it is possible that the colistin resistance mechanism in this study is due to mutations in chromosomal resistance genes. Although the mechanism of tigecycline resistance has not been fully elucidated, the tigecycline resistance rate increased from $19 \%$ to $36 \%$ with the increase in the $\operatorname{tet}(A)$ gene. This result suggests that the tetracycline resistance protein is involved in tigecycline resistance of CPKP isolates. Resistance to CST and TGC has been on the rise because these antibiotics are regularly prescribed due to the scarcity of treatment options for CPE $(5,42-44)$. A report stating that $45 \%$ of $298 \mathrm{~K}$. pneumoniae isolated at an Indian tertiary hospital between 2013 and 2017 were XDR and 9\% were PDR is alarming
(Mohapatra et al., 2018). Unfortunately, Mohapatra et al. (2018) do not mention the molecular epidemiology of XDR and PDR strains, but the possibility that these isolates are spreading from India and neighboring developing countries is plausible and worth investigating further.

Outbreaks involving the ST147 clone have been reported worldwide (Guo et al., 2016; Hamzaoui et al., 2018; Protonotariou et al., 2018). In Greece, the ST147 clone co-producing KPC2 and VIM-1 was isolated from 25 patients at a tertiary teaching hospital, and 17 of these patients developed bloodstream infections (mortality rate $=48 \%$ ) (Protonotariou et al., 2018). Nahid et al. (2017) provided insight into the emergence of ST147 PDR clone and the rapid global spreading of this high-risk clone. ST147 has been commonly reported among Indian isolates and has been frequently associated with NDM-1 and OXA48-like 


\section{$\square$ Multidrug resistant $\square$ Extensively drug resistant $\square$ Pandrug resistant}

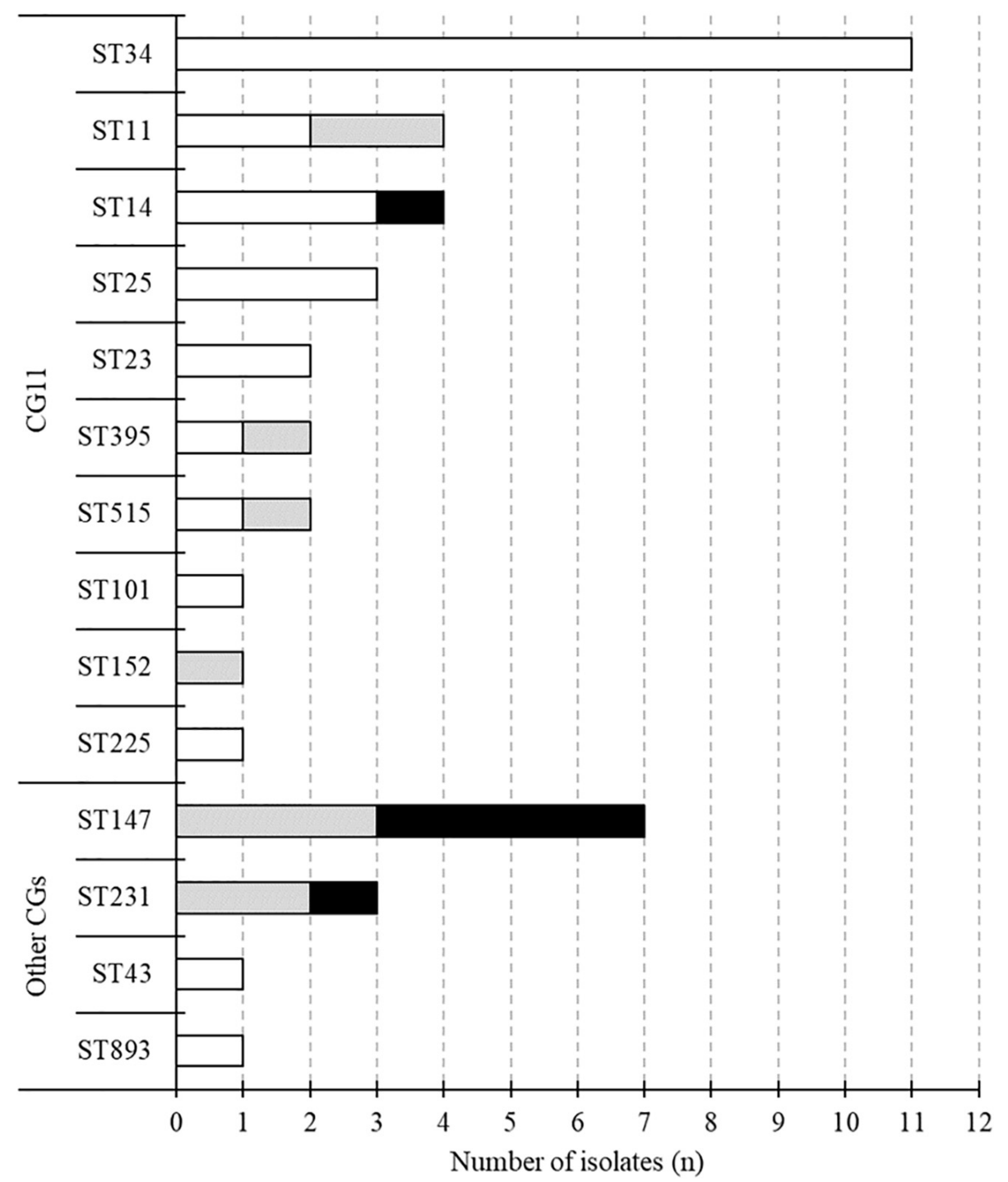

FIGURE 3 | Classification of multidrug resistance type of each STs.

carbapenemases as observed from the present study (Pragasam et al., 2017). Indeed, we showed that all ST147 clones were $\mathrm{XDR}$ and PDR strains harboring various resistance genes in Bangladesh. In addition, the ST147 clone was previously only isolated in the hospital environment, but a strain of ST147 that co-produces NDM-9 and CTX-M-15 has been isolated from wastewater in Switzerland (Nüesch-Inderbinen et al., 2018). This suggests that these PDR strains may already be present in our public health and are increasingly spreading under the surface. Therefore, ST147 will increasingly spread as a One Health infectious disease, and its dissemination may carry higher risks than other international high-risk clones such as ST11, ST15, and ST258.
In recent years, new antibacterial agents against carbapenemase-producing bacteria have been developed one after another and are expected to greatly contribute to the treatment of CPO infection (Ito et al., 2016). However, these new antimicrobials are expensive for some time after approval and are difficult to be readily available in developing countries. As another treatment option for CPO infections, a combination therapy by existing antibiotics is expected. We reported the efficacy of 136 pattern of combination therapies for five types of CPO and non-CPO (Okanda and Matsumoto, 2020). This report mainly suggests that the combined use of protein synthesis inhibitors and cell wall synthesis inhibitor is useful. However, CPOs isolated in South Asia often carry 16SRMTase, 
and there is concern that combinations using aminoglycosides are ineffective due to their high MIC values for aminoglycosides. Since $91 \%$ of CPKP isolates are AMK-high-resistant strains (MIC $\geq 512 \mu \mathrm{g} / \mathrm{ml}$ ) harboring 16SRMTase in this study, the effect of combination therapy using aminoglycosides cannot be expected. In our report, it is suggested that the combined use of BPM or IPM with MIN or TGC is effective to AMKhigh-resistant strains (Okanda and Matsumoto, 2020). This combination therapy may be effective for some PDR-CPKP isolates in Bangladesh, which are not highly resistant to these antimicrobials. However, as the MIC of the antibiotics used in combination increases, the effect of the combination is limited. Therefore, the increase in PDR strains and in MIC value are particularly serious problems for developing countries.

\section{CONCLUSION}

Spread of CPKP isolates and PDR strain in Bangladesh warrants the need for the intensive surveillance of AMR and the implementation of an efficient infection control program in developing countries for the management of such infections.

\section{DATA AVAILABILITY STATEMENT}

The original contributions presented in the study are publicly available. This data can be found here: [https://www.ddbj.nig.

\section{REFERENCES}

Al-Marzooq, F., Ngeow, Y. F., and Tay, S. T. (2015). Emergence of Klebsiella pneumoniae producing dual carbapenemases (NDM-1 and OXA-232) and 16S rRNA methylase (armA) isolated from a Malaysian patient returning from India. Int. J. Antimicrob. Agents. 45, 445-446. doi: 10.1016/j.ijantimicag.2014. 12.013

Bonomo, R. A., Burd, E. M., and Conly, J. (2009). Carbapenemase-producing organisms: a global scourge. Clin. Infect. Dis. 66, 1290-1297. doi: 10.1093/cid/ cix 893

Clinical and Laboratory Standards Institute (CLSI) (2020). Performance Standards for Antimicrobial Susceptibility Testing; M100, 30th Edn. Available online at: https://clsi.org/standards/products/free-resources/access- our-free-resources/ (accessed on 2020).

Diancourt, L., Passet, V., Verhoef, J., Grimont, P. A., and Brisse, S. (2009). Multilocus sequence typing of Klebsiella pneumoniae nosocomial isolates. J. Clin. Microbiol. 43, 4178-4182. doi: 10.1128/jcm.43.8.4178-4182.2005

Feil, E. J., Li, B. C., Aanensen, D. M., Hanage, W. P., and Spratt, B. G. (2004). eBURST: inferring patterns of evolutionary descent among clusters of related bacterial genotypes from multilocus sequence typing data. J. Bacteriol. 186, 1518-1530. doi: 10.1128/jb.186.5.1518-1530.2004

Fournier, S., Desenfant, L., Monteil, C., Nion-Huang, M., Richard, C., and Jarlier, V. (2018). Efficiency of different control measures for preventing carbapenemaseproducing enterobacteria and glycopeptide-resistant Enterococcus faecium outbreaks: a 6-year prospective study in a French multihospital institution, January 2010 to December 2015. Euro Surveill. 23, 17-00078.

Guo, L., An, J., Ma, Y., Ye, L., Luo, Y., Tao, C., et al. (2016). Nosocomial outbreak of OXA-48-producing Klebsiella pneumoniae in a Chinese hospital: clonal transmission of ST147 and ST383. PLoS One 11:e0160754. doi: 10.1371/journal. pone. 0160754

Hamzaoui, Z., Ocampo-Sosa, A., Maamar, E., Fernandez Martinez, M., Ferjani, S., Hammami, S., et al. (2018). An outbreak of NDM-1-producing Klebsiella pneumoniae, associated with OmpK35 and OmpK36 porin loss in Tunisia. Microb. Drug Resist. 24, 1137-1147. doi: 10.1089/mdr.2017.0165 ac.jp/index.html/ accession numbers: JN676828, KF240808, JN676878, JN676879, AF299299, KP050489, KF055402, KP772120, KP772206, KY418157, EF406115, KU985243, HQ267531, JX442976, AF227505, KJ511462, MK469978, MK412918, MK105834, AY220558, KY402263, JQ808129, CP052141, CP052141, EF682133, GQ438249, KX580704, MK043329, KU603636, KU603660, KY658724, JN108890, and AB759690].

\section{AUTHOR CONTRIBUTIONS}

TO: conceptualization, data curation, methodology, software, supervision, validation, visualization, writing - original draft, and writing - reviewing and editing. TO and TK: formal analysis. $\mathrm{AH}, \mathrm{HT}, \mathrm{TM}$, and $\mathrm{SN}$ : funding acquisition. TO, $\mathrm{AH}$, $\mathrm{AI}$, and $\mathrm{QH}$ : investigation. TO and TM: project administration. All authors contributed to the article and approved the submitted version.

\section{SUPPLEMENTARY MATERIAL}

The Supplementary Material for this article can be found online at: https://www.frontiersin.org/articles/10.3389/fmicb. 2020.612020/full\#supplementary-material

Hasan, M. J., and Rabbani, R. (2019). The need for adequate research data on carbapenem use and resistance in Bangladesh. Lancet Infect. Dis. 19:811. doi: 10.1016/s1473-3099(19)30353-6

Ito, A., Nishikawa, T., Matsumoto, S., Yoshizawa, H., Sato, T., Nakamura, R., et al. (2016). Siderophore cephalosporin cefiderocol utilizes ferric iron transporter systems for antibacterial activity against Pseudomonas aeruginosa. Antimicrob. Agents Chemother. 60, 7396-7401.

Macesic, N., Nelson, B., Mcconville, T. H., Giddins, M. J., Green, D. A., Stump, S., et al. (2020). Emergence of polymyxin resistance in clinical Klebsiella pneumoniae through diverse genetic adaptations: a genomic, retrospective cohort study. Clin. Infect. Dis. 70, 2084-2091. doi: 10.1093/cid/ ciz623

Magiorakos, A. P., Srinivasan, A., Carey, R. B., Carmeli, Y., Falagas, M. E., Giske, C. G., et al. (2012). Multidrug-resistant, extensively drug-resistant and pandrug-resistant bacteria: an international expert proposal for interim standard definitions for acquired resistance. Clin. Microbiol. Infect. 18, 268-281. doi: 10.1111/j.1469-0691.2011.03570.x

Mathers, A. J., Peirano, G., and Pitout, J. D. (2015). The role of epidemic resistance plasmids and international high-risk clones in the spread of multidrug-resistant Enterobacteriaceae. Clin. Microbiol. Rev. 28, 565-591. doi: 10.1128/CMR.00 116-14

Mohapatra, D. P., Debata, N. K., and Singh, S. K. (2018). Extensively drug-resistant and pan-drug resistant Gram-negative bacteria in a tertiary-care hospital in Eastern India: a 4 year retrospective study. J. Glob. Antimicrob. Resist. 15, 246-249. doi: 10.1016/j.jgar.2018.08.010

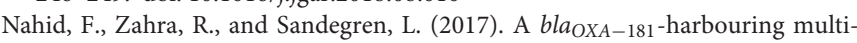
resistant ST147 Klebsiella pneumoniae isolate from Pakistan that represent an intermediate stage towards pan-drug resistance. PLoS One 12:e0189438. doi: 10.1371/journal.pone.0189438

Nüesch-Inderbinen, M., Zurfluh, K., Stevens, M. J. A., and Stephan, R. (2018). Complete and assembled genome sequence of an NDM-9- and CTX-M-15-producing Klebsiella pneumoniae ST147 wastewater isolate from Switzerland. J. Glob. Antimicrob. Resist. 13, 53-54. doi: 10.1016/j.jgar.2018. 03.001 
Okanda, T., Haque, A., Ehara, T., Huda, Q., Ohkusu, K., Miah, R. A., et al. (2018). Characteristics of resistance mechanisms and molecular epidemiology of fluoroquinolone-nonsusceptible Salmonella enterica serovar typhi and paratyphi a isolates from a tertiary hospital in Dhaka, Bangladesh. Microb. Drug Resist. 24, 1460-1465. doi: 10.1089/mdr.2018.0039

Okanda, T., and Matsumoto, T. (2020). In vitro effect of an antimicrobial combination therapy without colistin and tigecycline for CPE and non-CPE. J. Infect. Chemother. 26, 322-330. doi: 10.1016/j.jiac.2019.12.004

O'neill, J. (2016). Tackling Drug-Resistant Infections Globally: Final Report and Recommendations. The Review on Antimicrobial Resistance. Available online at: https://amr-review.org/ (accessed on 2016).

Papadimitriou-Olivgeris, M., Bartzavali, C., Spyropoulou, A., Lambropoulou, A., Sioulas, N., Vamvakopoulou, S., et al. (2018). Molecular epidemiology and risk factors for colistin- or tigecycline-resistant carbapenemase-producing Klebsiella pneumoniae bloodstream infection in critically ill patients during a 7-year period. Diagn. Microbiol. Infect. Dis. 92, 235-240. doi: 10.1016/j.diagmicrobio. 2018.06.001

Pragasam, A. K., Shankar, C., Veeraraghavan, B., Biswas, I., Nabarro, L. E., Inbanathan, F. Y., et al. (2017). Molecular mechanisms of colistin resistance in Klebsiella pneumoniae causing bacteremia from India-A first report. Front. Microbiol. 7:2135. doi: 10.3389/fmicb.2016.02135

Protonotariou, E., Poulou, A., and Politi, L. (2018). Hospital outbreak due to a Klebsiella pneumoniae ST147 clonal strain co-producing KPC-2 and VIM-1 carbapenemases in a tertiary teaching hospital in Northern Greece. Int. J. Antimicrob. Agents 52, 331-337. doi: 10.1016/j.ijantimicag.2018. 04.004

Conflict of Interest: The authors declare that the research was conducted in the absence of any commercial or financial relationships that could be construed as a potential conflict of interest.

Copyright (c) 2021 Okanda, Haque, Koshikawa, Islam, Huda, Takemura, Matsumoto and Nakamura. This is an open-access article distributed under the terms of the Creative Commons Attribution License (CC BY). The use, distribution or reproduction in other forums is permitted, provided the original author(s) and the copyright owner(s) are credited and that the original publication in this journal is cited, in accordance with accepted academic practice. No use, distribution or reproduction is permitted which does not comply with these terms. 